\title{
Efectos del fuego en el arbolado de un bosque tropical de pino y en el de una selva baja caducifolia en Villaflores, Chiapas
}

\author{
Fire effects on the trees of a tropical pine forest and a tropical dry forest at \\ Villaflores, Chiapas, Mexico
}

\begin{abstract}
Dante Arturo Rodríguez Trejo ${ }^{\mathrm{I}}$, Pedro Martínez Muñoz ${ }^{\mathrm{II}}$, Pedro Jerónimo Martínez Lara ${ }^{\mathrm{III}}$
\end{abstract}
\section{Resumen}

Debido al aumento global de incendios forestales que se espera con el cambio climático, es necesario entender mejor sus efectos y la ecología del fuego en diferentes ecosistemas. En el municipio de Villaflores, Chiapas, se hacen esfuerzos en manejo integral del fuego. En ese lugar se estudió un bosque de Pinus oocarpa Schiede y una selva baja caducifolia, incendiados 6 y 18 meses antes, respectivamente. Los objetivos fueron: modelar la probabilidad de mortalidad y de rebrotación del primero y estudiar la mortalidad, composición y adaptaciones al fuego en la segunda. En ambos bosques se registraron variables dasométricas, de severidad del fuego y se calcularon valores de importancia. Del pinar se obtuvieron modelos logísticos para estimar probabilidades de mortalidad y de rebrotación, que confirman adaptaciones al fuego y características de los árboles que les permiten sobrevivir. Valores altos en: altura, diámetros, altura a la base de las copas y grosor de corteza, reducen la probabilidad de mortalidad. Mayor altura de cicatriz sobre el tronco, la incrementa. Aunque se trata de una especie adaptada al fuego, con corteza gruesa, recuperación de copa mediante rebrotes epicórmicos, rebrotación en la base del tronco y regeneración, el incendio fue severo, con una mortalidad de $48,8 \%$ y altura media de la cicatriz del fuego sobre el tronco de $1,5 \mathrm{~m} \pm 1,3 \mathrm{~m}$. En la selva baja el incendio no fue severo, con mortalidad de $5 \%$, se hallaron 37 especies arbóreas y hubo 28 de ellas con adaptaciones al fuego, como: corteza gruesa, latencia física en semilla o pireno del fruto, regeneración y rebrotación. La abundancia de especies con adaptaciones al fuego, deja ver que históricamente los incendios eliminaron especies sensibles y que esta selva, alterada, tiene más especies adaptadas al fuego que las que se pensaba y la ubica como influenciada por el fuego.

Palabras clave: Ecología del fuego; Pinus oocarpa; Pinar; Selva baja

\begin{abstract}
It is expected an increase of forest fires world-wide because of global warming. So is important the study of fire ecology and fire effects in different ecosystems. In Villaflores, Chiapas, Mexico, are conducted efforts towards the integral fire management. There was studied a Pinus oocarpa Schiede forest, as well as a tropical dry forest, affected by forest fires 6 and 18 months before, respectively. The objectives were: To model the probability of mortality and probability of resprouting in the former, and to study mortality, composition and fire-traits in the later. In both forests were recorded dasometric and fire severity variables, and calculated importance values. For the pine forest were obtained logistic models to estimate probabilities of mortality and basal resprouting. The obtained models confirm fire adaptations and tree-traits that facilitate tree survival. High values in tree height, basal diameter, diameter at breast height, height to the base of crown and bark thickness, reduce probability of mortality. But high levels of fire trunk scar height, increase it. Despite this species is fire-adapted with thick bark, epicormic resprouts that restore the crown, basal resprouting and regeneration; the forest fire was severe, pine mortality reached $48,8 \%$, and the mean trunk scar height was $1,5 \mathrm{~m} \pm 1,3 \mathrm{~m}$. In the tropical dry forest the fire was not severe, with a mortality of $5 \%$, and were recorded 37 tree species, 28 of them with several fire-traits, such as: thick bark, physical dormancy in seeds or pyrene in the fruit, regeneration and resprouting. The prevalence of fire-adapted species, shows the possibility that they are the result of historic fires that eliminated sensitive species and that in this altered tropical dry forest there are more fire-adapted species than thought, so is fire-influenced.
\end{abstract}

Keywords: Fire ecology; Pine forest; Pinus oocarpa; Tropical decidouos forest

Ingeniero Agrónomo, Ph. D., Profesor-Investigador de la División de Ciencias Forestales, Universidad Autónoma Chapingo, km. 38.5 carretera México-Texcoco, Chapingo, Edo. de Méx., C.P. 56230, México. dantearturo@yahoo.com (ORCID: 0000-0002-1407-8365)

II Técnico Forestal, Director de Biomasa, A. C., segunda Norte no. 62, entre Tercera y Cuarta Oriente, Barrio Matzumóm, Villaflores, Chiapas, C. P. 30470. pmtz29@hotmail.com (ORCID: 0000-0001-7675-4392)

III Estudiante, Universidad de Ciencias y Artes de Chiapas, Sur Poniente 1460, Colonia Centro, Tuxtla Gutiérrez, Chiapas, México, C. P. 29000. pmtz_19@hotmail.com (ORCID: 0000-0001-6221-5777) 


\section{Introducción}

El cambio climático está incrementando frecuencia, intensidad y severidad de los incendios en el planeta (SHLISKY et al., 2007). La clasificación de ecosistemas con base en su relación con el fuego (HARDESTY; MYERS; FULKS, 2005), aplica para México (RODRÍGUEZTREJO, 2008). En ella hay cuatro categorías de ecosistemas: mantenidos por el fuego, sensibles al fuego, influenciados por el fuego e independientes del fuego. En los ecosistemas mantenidos por el fuego, como los pinares, éste cumple una función ecológica y contribuye a mantener la etapa sucesional presente. Las especies están adaptadas al fuego. En los ecosistemas sensibles al fuego, como las selvas, hay menos adaptaciones a ese factor, se causa elevada mortalidad y tomará décadas o siglos a la sucesión ecológica restablecer la composición y estructura presentes antes del incendio. En los ecosistemas influenciados por el fuego, existen tanto especies adaptadas como no adaptadas a este factor ecológico, puede tratarse de ecotonos. Finalmente, en los ecosistemas independientes del fuego, no hay condiciones que permitan a los incendios propagarse; por ejemplo, debido a la falta de continuidad horizontal en los combustibles forestales de las zonas más áridas.

La gran mayoría de los pinos mexicanos muestran adaptaciones al fuego y Pinus oocarpa Schiede, el pino tropical más ampliamente distribuido en México, no es la excepción, pues se regenera bien en áreas incendiadas, tiene una corteza gruesa que protege su cambium vascular del calor del fuego, emite rebrotes epicórmicos y de la base, siempre que las yemas no mueran (RODRÍGUEZ-TREJO; FULÉ, 2003; RODRÍGUEZ-TREJO, 2014).

La estimación de la probabilidad de mortalidad de los árboles por efecto del fuego a diferentes intensidades y severidades, reviste interés tanto para hacer predicciones de la mortalidad en áreas afectadas por incendios forestales, como para tener una base de información que permita estimar que la mortalidad del arbolado luego de una quema prescrita, será nula o pequeña, según los objetivos, así como las características de la quema (gobernada por combustibles, tiempo atmosférico y topografía), y las del arbolado. Un esfuerzo relevante de modelación de probabilidad de mortalidad por fuego en coníferas del oeste de Estados Unidos, es el de Hood et al. (2007). En el caso de México, se comenzó este tipo de trabajos en Pinus hartwegii Lindl. (RODRÍGUEZ-TREJO et al., 2007), pero también se han llevado a cabo en especies como Quercus crassifolia Bonpl. (JUÁREZ; RODRÍGUEZ-TREJO; MYERS, 2012), entre otras pocas. Las diferentes características de los incendios en distintas áreas de distribución de cada especie, hacen necesarios más estudios de este tipo no solo para otras especies, sino para las mismas en diferentes escenarios de fuego y de distribución natural, para entender mejor las características de los árboles, del ambiente y del fuego, que moldean la supervivencia.

En general se considera que las especies arbóreas tropicales típicas de selvas altas y medianas son sensibles al fuego (SHLISKY et al., 2007), excepto por las que al degradarse la selva quedan conformando sabanas. Sin embargo, en el caso de las selvas bajas la situación no es clara. Existe evidencia de que algunas selvas bajas caducifolias o subcaducifolias, con abundancia de árboles de la familia de las leguminosas y con pastos como uno de sus componentes naturales en el sotobosque, tienen más posibilidades de estar relacionadas con el fuego (RODRÍGUEZTREJO, 2014). En el ejido Melchor Ocampo, municipio de Villaflores, Chiapas, se observó una selva baja caducifolia afectada por incendio forestal. Contra lo que se esperaba, la mortalidad no fue alta. De ahí el interés de estudiar la composición de tal selva.

En el municipio de Villaflores, se hacen esfuerzos hacia el manejo integral del fuego. De acuerdo con Rodríguez-Trejo (2000), tal concepto involucra el fundir el uso del fuego con fines ecológicos y bases científicas, con el uso tradicional del fuego para que la gente del campo lo siga empleando, pero más juiciosamente, así como con la prevención y combate de incendios forestales no deseados.

Un programa de quemas prescritas, para vegetación mantenida por el fuego, como los pinares, requiere de fundamentos sólidos en diversos aspectos; entre ellos, conocer la resistencia, 
tolerancia o sensibilidad al fuego de las diferentes especies forestales en los ecosistemas bajo manejo. De ahí la relevancia de estudiar este aspecto.

Los objetivos del presente trabajo, son: a) establecer variables de los árboles, de severidad del fuego y modelos que permitan estimar las probabilidades de mortalidad y rebrotación postfuego para Pinus oocarpa, y b) estudiar la composición, mortalidad y adaptaciones al fuego de las especies arbóreas en una selva baja caducifolia afectada por incendio forestal.

Como hipótesis se establece que en la medida que los pinos tengan mayores dimensiones, menor será su probabilidad de mortalidad, gracias a sus adaptaciones al fuego; en tanto que para la selva baja se espera hallar una prevalencia de especies no adaptadas al fuego, y que el incendio fue poco severo como para originar mortalidad importante en sus árboles.

\section{Materiales y métodos}

Para el trabajo de campo, se muestreó el arbolado en dos áreas incendiadas. La primera, en el ejido Sombra de la Selva, Mpio. de Villaflores, en la Reserva de la Biosfera La Sepultura, Chiapas, un bosque de Pinus oocarpa afectado (40 ha) por incendio forestal 6 meses antes del muestreo $\left(16^{\circ} 19^{\prime} 53^{\prime \prime} \mathrm{N}\right.$ y $\left.93^{\circ} 37^{\prime} 40^{\prime \prime} \mathrm{O}\right)$. El clima es cálido subhúmedo y los suelos son regosoles. En octubre de 2016 fueron levantados ahí 14 sitios al azar de $400 \mathrm{~m}^{2}$ cada uno. La segunda área, se enclava en el ejido Melchor Ocampo, Mpio de Villaflores, donde un incendio forestal (110 ha) $\left(16^{\circ} 19^{\prime} 58^{\prime \prime} \mathrm{N}\right.$ y $\left.93^{\circ} 29^{\prime} 57^{\prime} \mathrm{O}\right)$ afectó una selva baja caducifolia en 2015 , un año y medio antes del muestreo. El clima es cálido subhúmedo y los suelos son regosoles. En diciembre de 2016 fueron levantados 21 sitios de muestreo de $100 \mathrm{~m}^{2}$ cada uno, ubicados al azar.

En cada sitio fueron registradas variables dasométricas en árboles con $2 \mathrm{~m}$ o más de altura: especie, diámetro en la base $(\mathrm{cm})$, diámetro normal $(\mathrm{cm})$, altura total $(\mathrm{m})$, altura a la base de la copa original $(\mathrm{m})$ y grosor de corteza $(\mathrm{mm})$. También fueron medidas variables relacionadas con la severidad del fuego o nivel de daño al arbolado: si el árbol estaba vivo o muerto, en el primer caso si contaba con rebrotes post-fuego, la altura de la cicatriz del fuego sobre el tronco (m) y el porcentaje de copa chamuscado. Esta última variable no se tomó en cuenta en la selva baja, por su carácter caducifolio. Se consideraron muertos a los árboles cuando carecían de follaje o si estaban todos necrosados de la copa, no mostraban rebrotación ni en la base del tronco ni en este ni en la copa o raíz y si el tronco tenía la madera seca al corte. La mortalidad se calculó dividiendo el número de árboles muertos entre la suma de árboles muertos más vivos y multiplicando el resultado por cien. Esta última se calculó para Pinus oocarpa en el bosque de pino y para todas las especies en general en la selva baja. Las mediciones se hicieron con regla, flexómetro, estadal graduado y clinómetro. Además, fueron registradas la o las especie(s) dominante(s) en la regeneración. La identificación de las especies se llevó a cabo usando principalmente las siguientes referencias: Villegas et al. (2000), Pennington y Sarukhán (2005), Ochoa, Pérez y Jiménez (2008), García y Linares (2013) y Miranda (2015a; 2015b). Las adaptaciones al fuego fueron consultadas, de las existentes para otras especies, en Miller (2000), RodríguezTrejo (2014) y Scott et al. (2014).

Para el trabajo de gabinete, la información de campo fue recabada y sistematizada en hojas electrónicas (prog rama Excel, Office $\left.{ }^{\circledR}\right)$. Se calculó el porcentaje de valor de importancia (\%VI) para las especies arbóreas en bosque de pino y selva baja. Los valores de importancia de cada especie fueron obtenidos sumando la frecuencia relativa (FR, frecuencia en los sitios de muestreo de cada especie entre la suma de las frecuencias de todas las especies) más la dominancia relativa (DoR, área basal de cada especie entre la suma de las áreas basales de todas las especies) más la densidad relativa (DeR, densidad de cada especie entre la suma de las densidades de todas las especies) (ODUM; BARRETT, 2006). Conformada la base de datos para el bosque de pino, fue utilizada para alimentar al sistema SAS ${ }^{\circledR}$ (Statistical Analysis System; Sistema de Análisis Estadístico) v. 9,4 para microcomputadoras. Con la ayuda de dicho programa, fueron corridas regresiones logísticas (HOSMER JUNIOR; LEMESHOW; STURDIVANT, 2013). Tales regresiones estiman 
la probabilidad de mortalidad o de rebrotación a partir de variables explicativas. Estas últimas fueron variables dasométricas y de severidad del fuego. Las variables explicativas fueron corridas individualmente y luego en combinaciones, hasta incluir todas. De los modelos obtenidos, fueron seleccionados los más robustos (con todas sus variables significativas, $p<0,05$ e intervalo de confianza que no incluyera el número 1, el cual implicaría que la probabilidad de ocurrencia es igual a la de no ocurrencia). También se buscó que hubiera una alta concordancia ( $>50 \%)$ y se llevó a cabo una prueba de bondad de Ajuste (la de Hosmer y Lemeshow). En este último caso, se buscó que no resultara significativa $(\mathrm{p}>0,05)$.

\section{Resultados y discusión}

\section{Bosque de Pinus oocarpa}

En total fueron muestreados 151 árboles. De ellos, 82,1\% fueron Pinus oocarpa, 13,9\% encinos (Quercus spp.), 2\% nanches (Byrsonima crassifolia) y el resto (2\%) de otras especies. Los $\% \mathrm{VI}$ previos al fuego, alcanzaron, respectivamente: 71,9, 21,5, 4,6 y 2,0. Los \%VI post-fuego, en el mismo orden, fueron: 72,7, 22,1, 2,1 y 3,1. La masa de pinos era joven, con altura promedio de $8 \mathrm{~m}$ y diámetro normal medio igual a $13 \mathrm{~cm}$. Sin embargo, había mucha variabilidad de tamaños, característica deseable para realizar el presente estudio (Tabla 1).

El incendio fue severo, pues la mortalidad del pino alcanzó $48,8 \%$ y $57,1 \%$ la de los encinos. Los promedios de altura de la cicatriz del fuego sobre el tronco y de chamuscado de copas alcanzaron 1,5 m y 68,7\%. Las características de la masa, antes y después del incendio, revelan que este causó mayor mortalidad en los individuos jóvenes y los de dimensiones pequeñas, dejando una masa residual menos densa, menos variable y de mayores dimensiones. La densidad media de la masa previa al incendio fue $219,6 \mathrm{ha}^{-1}\left( \pm 114 \mathrm{ha}^{-1}\right)$ y la posterior al fuego $112,5 \mathrm{ha}^{-1}\left( \pm 75.8 \mathrm{ha}^{-1}\right)$. De la misma forma, los individuos supervivientes muestran menores niveles de daño (Tabla 1).

Para la probabilidad de mortalidad $(\mathrm{Pm})$, resultaron significativas $(\mathrm{p}<0,05)$, con el intervalo de confianza sin incluir 1 , una alta concordancia $(>50 \%)$ y con $\mathrm{p}>0,05$ para la prueba de bondad de ajuste de Hosmer y Lemeshow, las variables y los modelos 1 a 12 :

Tabla 1 - Características dasométricas de Pinus oocarpa vivos, antes y después del fuego.

Table 1 - Dasometric characteristics of alive Pinus oocarpa, before and after the fire.

\begin{tabular}{lcccc}
\hline \multicolumn{1}{c}{ Variable } & Antes & Después & Máxima(o) & Mínima $(\mathbf{o})$ \\
\hline $\mathbf{A}(\mathbf{m})$ & $8,5 \pm 5,2$ & $11,0 \pm 5,2$ & 30 & 2 \\
$\mathbf{D B}(\mathbf{c m})$ & $16,5 \pm 12,6$ & $23,2 \pm 13,9$ & 91 & 3 \\
$\mathbf{D N}(\mathbf{c m})$ & $13,0 \pm 11,3$ & $19,0 \pm 12,3$ & 79 & 2 \\
$\mathbf{A C}(\mathbf{m})$ & $3,4 \pm 3,4$ & $4,8 \pm 4,0$ & 16 & 1 \\
$\mathbf{G C}(\mathbf{m m})$ & $11,5 \pm 6,7$ & $14,0 \pm 6,7$ & 30 & 2 \\
ACT $(\mathbf{m})$ & $1,5^{\star} \pm 1,2$ & $1,6 \pm 1,4$ & 10 & 0,2 \\
$\mathbf{C h}(\%)$ & $68,7^{\star} \pm 37,0$ & $38,8 \pm 29,0$ & 100 & 0 \\
\hline
\end{tabular}

Donde: $\mathrm{A}=$ altura; $\mathrm{DB}=$ diámetro a la base; $\mathrm{DN}=$ diámetro normal; $\mathrm{AC}=$ altura a la base de la copa; $\mathrm{GC}=$ grosor de corteza; $\mathrm{ACT}=$ altura de la cicatriz sobre el tronco; $\mathrm{Ch}=$ chamuscado de copas; Antes = antes del incendio (árboles vivos y muertos); Después = después de éste (árboles vivos). ${ }^{*}=$ No es previo al incendio en este caso, sino la inclusión de árboles vivos y muertos. 


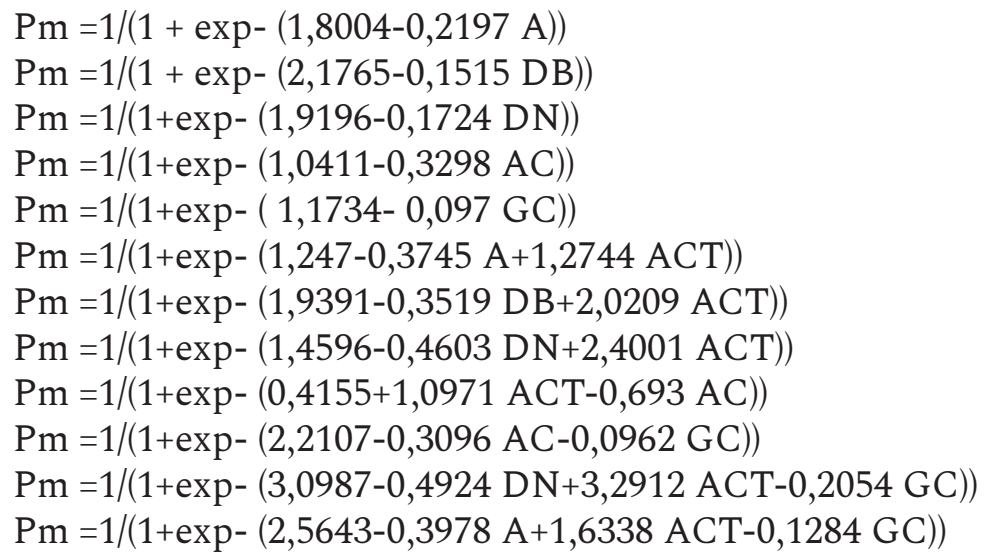

Las abreviaturas se dan al pie de la Tabla 1. Las Figuras 1 a 3, muestran las gráficas de algunos modelos.

Figura 1 - Probabilidad de mortalidad de Pinus oocarpa como función de: A) altura, B) diámetro normal, C) altura a la base de la copa y D) grosor de corteza. Modelos 1, 3, 4 y 5, respectivamente.

Figure 1 - Probability of fire-caused mortality of Pinus oocarpa as a function of: A) height, B) diameter at breast height, $C$ ) height to the base of crown, and D) bark thickness. Models 1, 3, 4 and 5 , respectively.

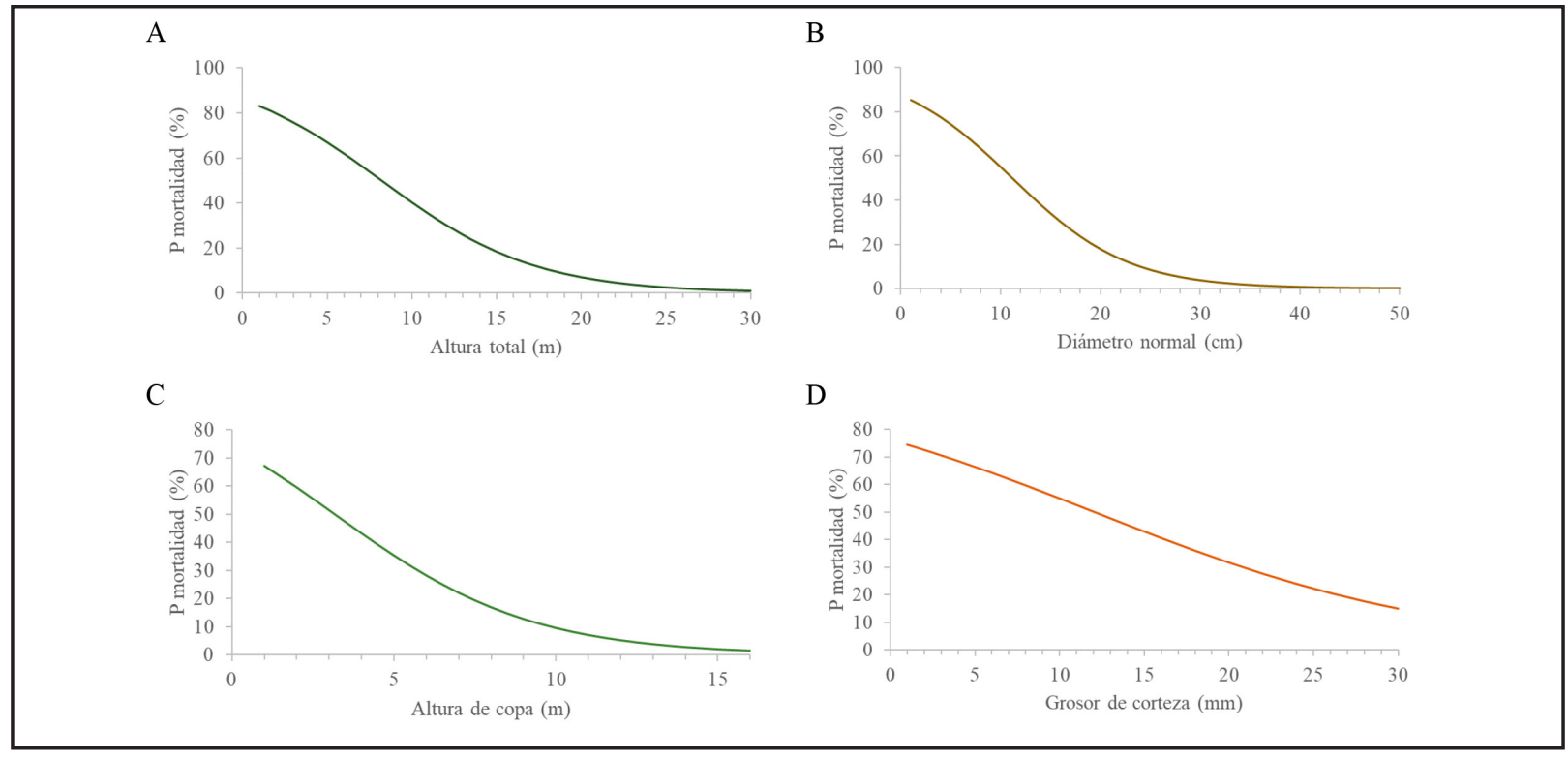

Fuente: Autores (2019)

Source: Authors (2019)

En la medida que un árbol es más alto, tendrá también una mayor parte de las copas fuera del alcance del fuego, es decir, del follaje o tejido para la fotosíntesis que permitirá la producción de carbohidratos para rebrotación de follaje y ramas de las partes dañadas. De igual forma, la yema apical y las de las ramas, se encuentran más elevadas (Figura 1A). De acuerdo con Miller (2000), conforme un árbol tiene mayor diámetro, tenderá a tener más gruesa su corteza y esta protegerá mejor al cambium vascular (Figura 1B).

El fuego puede afectar a la copa en distintas zonas, quemándola directamente, 
deshidratándola y matando a las yemas, deshidratándola, pero sin matar a las yemas y sometiéndola a temperaturas elevadas mas no letales para el follaje. En la medida que la base de la copa se encuentra a mayor altura, se reduce la posibilidad de que sea afectada por las altas temperaturas del fuego a cualquier nivel de daño. De igual forma, al incrementar la altura a la base de la copa, se reduce la continuidad vertical entre combustibles y se abate la posibilidad de que la copa arda entera en años muy secos, durante los cuales el contenido de humedad del follaje vivo también es bajo y crítico para el peligro de incendio de copas (Figura 1C).

Dado que el grosor de corteza es considerado una adaptación al fuego en ambientes con incendios sometidos a incendios frecuentes o relativamente frecuentes, conforme un árbol tiene mayor esta dimensión su cambium vascular estará más protegido de las elevadas temperaturas y será menos probable que resulte cinchado térmicamente por el fuego (MILLER, 2000). A menor grosor de corteza, mayor posibilidad de daño al cambium vascular y a los tejidos de conducción (alterando transporte de agua, nutrimentos y fotosintatos), con lo que aumenta la probabilidad de mortalidad (HOOD et al., 2018) (Figura 1D).

Figura 2 - Probabilidad de mortalidad de Pinus oocarpa por el incendio, en función de su altura y de la altura de cicatriz por el fuego sobre el tronco. Modelo 6.

Figure 2 - Probability of fire-caused mortality of Pinus oocarpa as a function of its height and the height of the fire-scar on trunk. Model 6.

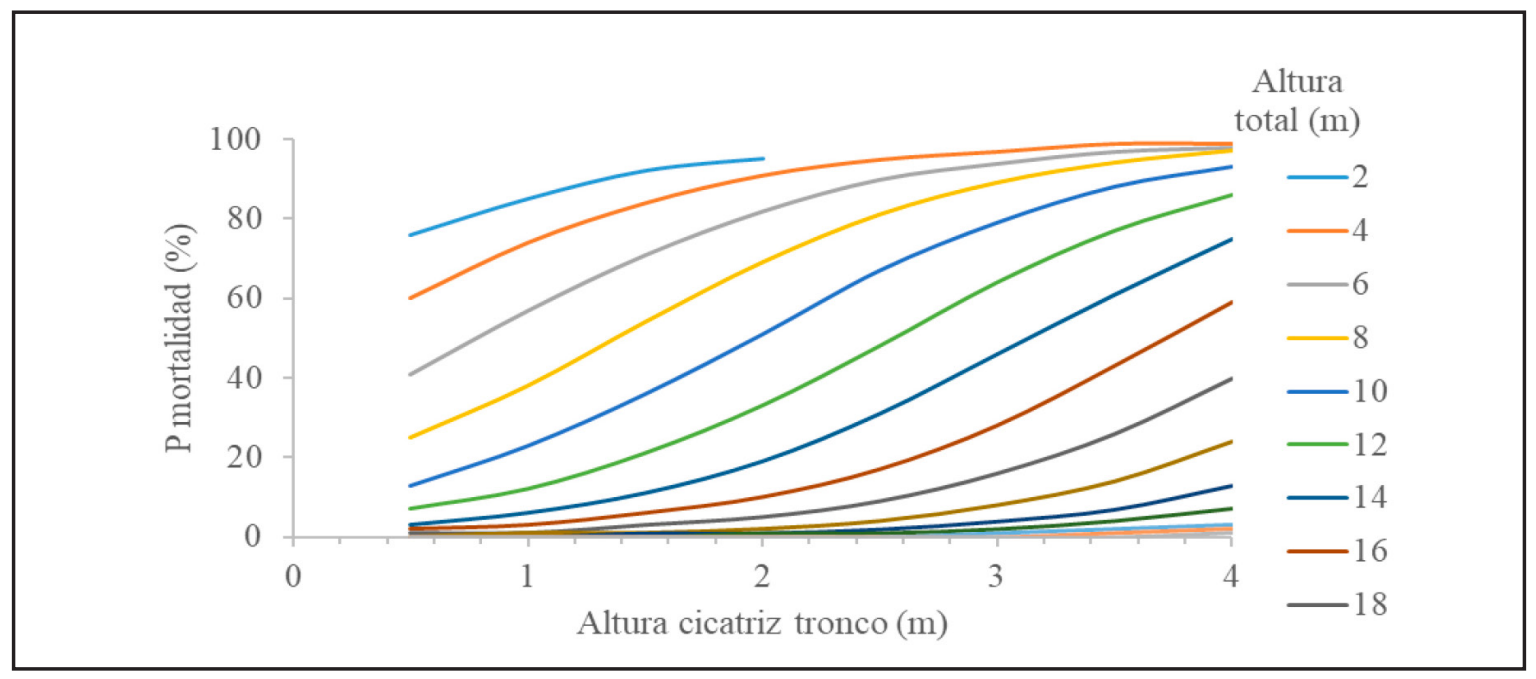

Fuente: Autores (2019)

Source: Authors (2019)

Si bien las mayores categorías de altura tienen una baja probabilidad de mortalidad, en cada una de sus curvas (Figura 2), la mayor altura de la cicatriz por el fuego sobre el tronco aumenta la probabilidad de mortalidad. El efecto es simple, conforme la citada cicatriz es más alta, el fuego habrá causado mayor daño al tronco, al cambium (a pesar del grosor de corteza), al follaje y al tejido de conducción. Hasta la categoría de altura de $14 \mathrm{~m}$, se tienen altas probabilidades de mortalidad incluso con bajas alturas de cicatriz por fuego sobre el tronco, debido a la poca altura. Sin embargo, a partir de los $16 \mathrm{~m}$ de altura, se observa que alrededor de $2,5 \mathrm{~m}$ de altura de cicatriz sobre el tronco, aumenta más la probabilidad de mortalidad. La Figura 3, muestra el aumento de la probabilidad de mortalidad al reducir diámetro y grosor de corteza y al incrementar la altura de la cicatriz sobre el tronco, simultáneamente.

El presente estudio evaluó probabilidad de mortalidad en un área de incendio forestal, mucho más intenso de lo que se alcanza en condiciones ideales de quema prescrita. Sin embargo, 
los indicadores de mortalidad también son de utilidad para estimarla luego de quemas prescritas, donde generalmente se busca que sea nula o mínima. De tal forma se pueden alcanzar objetivos de quemas prescritas, como la reducción de la carga de combustibles y del peligro de incendio, rebrotación de pastos para pastoreo y fauna, regeneración del bosque y mantenimiento del pinar, entre otros, pero con mínima o nula afectación al arbolado.

Se obtuvieron cinco modelos significativos (13 a 17, Figura 4) para la probabilidad de rebrotación $(\mathrm{Pr})$ de Pinus oocarpa $(\mathrm{p}<0,05$, intervalo de confianza sin 1 , concordancia $>50 \%$ y prueba de bondad de ajuste de Hosmer y Lemeshow con $\mathrm{p}>0,05)$ :

$\begin{array}{ll}\operatorname{Pr}=1 /(1+\exp -(0,5342-0,1775 \mathrm{~A})) & {[13]} \\ \operatorname{Pr}=1 /(1+\exp -(1,2013-0,16 \mathrm{DB})) & {[14]} \\ \operatorname{Pr}=1 /(1+\exp -(0,8987-0,1784 \mathrm{DN})) & {[15]} \\ \operatorname{Pr}=1 /(1+\exp -(0,8239-0,6569 \mathrm{AC})) & {[16]} \\ \operatorname{Pr}=1 /(1+\exp -(-2,1955+0,0189 \mathrm{Ch})) & {[17]}\end{array}$

Figura 3 - Probabilidad de mortalidad de Pinus oocarpa con diferentes diámetros normales, para distintas alturas de cicatriz sobre el tronco y grosores de corteza. Nótese que en el último gráfico la escala de probabilidades de mortalidad está en X (10) $)^{-3}$. Modelo 11.

Figure 3 - Probability of mortality of Pinus oocarpa, for different diameters at breast height, heights of fire-scars on trunk and bark thicknesses. Note that in the last graph the scale of probabilities is in X (10)-3. Model 11.

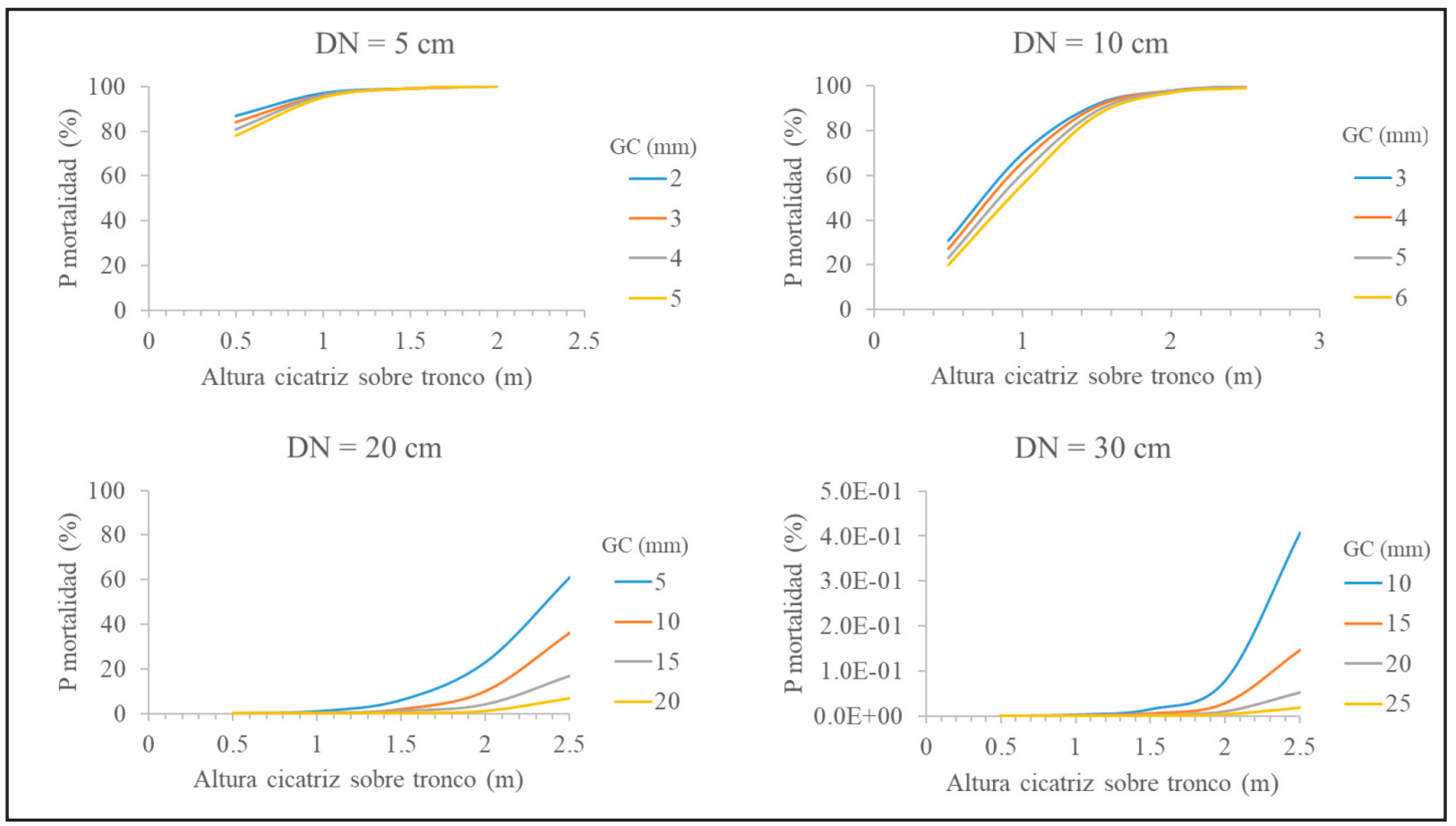

Fuente: Autores (2019)

Source: Authors (2019)

Se observó rebrotación en la base de $33 \%$ de los pinos muestreados, los más jóvenes supervivientes con alta afectación de copas. Tales rebrotes, al cabo de 6 meses del incendio forestal, habían alcanzado $40 \mathrm{~cm}$ como longitud media. En la propagación vegetativa, la juvenilidad de individuos o de ramas es un requisito para tener un buen prendimiento (HARTMANN et al., 2011). De manera similar, el tejido joven dañado a niveles no letales se recupera del daño y rebrota mejor. De ahí 
la relación inversa entre la altura y el diámetro con la probabilidad de rebrotación en la especie bajo estudio. Por otra parte, a mayor nivel de afectación de la copa, los árboles supervivientes tenderán a rebrotar mejor. De acuerdo con Miller (2000), este fenómeno puede relacionarse con la eliminación de auxinas en yemas apicales y follaje próximo junto con parte de la copa, ya que dicha fitohormona es inhibidora de la rebrotación. Conforme las auxinas desaparecen de una mayor porción de la copa, será más posible que el árbol rebrote, gracias a las citocininas en la raíz que son enviadas a las yemas supervivientes, en la base del árbol, por ejemplo, y a reservas de carbohidratos con que cuenta, para comenzar a recuperar superficie fotosintética (ver gráficas de algunos modelos en la Figura 4).

\section{Selva baja}

En el área de estudio, los árboles tuvieron alturas de 2 a $15 \mathrm{~m}$ (media=5,74 $\mathrm{m} \pm 2,90 \mathrm{~m}$ ), así como diámetros normales entre 1 y $31 \mathrm{~cm}$ (media $=7,4 \mathrm{~cm} \pm 5,1 \mathrm{~cm}$ ). El fuego fue de baja intensidad y severidad, ya que casi en ningún árbol se apreció la cicatriz que deja el fuego sobre el tronco y la supervivencia fue alta, $95 \%$.

Figura 4 - Probabilidad de rebrotación post-fuego de Pinus oocarpa como función de: A) altura total, B) porcentaje de chamuscado de copas. Modelos 13 y 17.

Figure 4 - Post-fire resprouting probability of Pinus oocarpa as a function of: A) total height, B) percentage of crown scorch. Models 13 and 17.

A

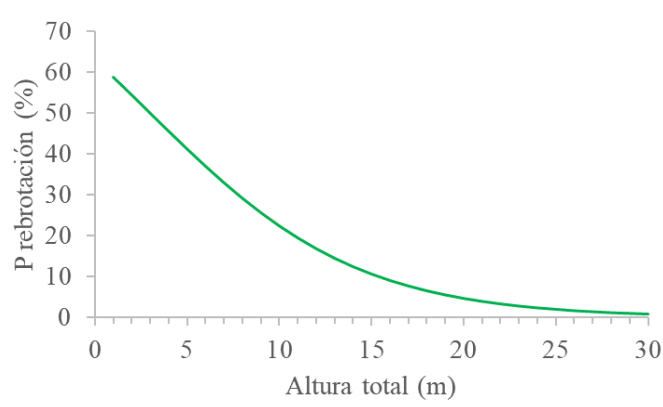

B

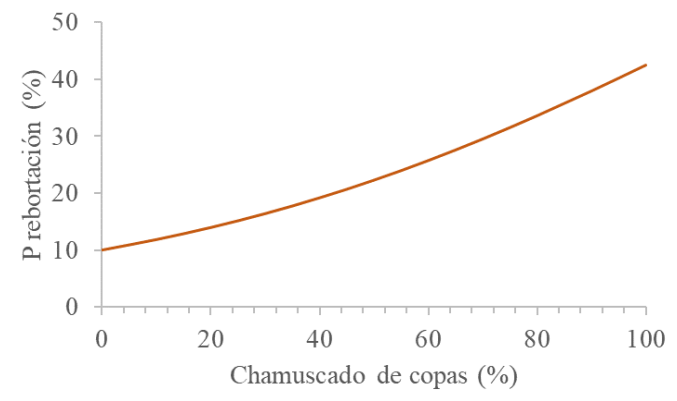

Fuente: Autores (2019)

Source: Authors (2019)

En el muestreo se registró una riqueza de 37 especies arbóreas. Los mayores \%VI (> 5) los tuvieron Heliocarpus reticulatus, Bursera bipinnata, Liabum sublobatum, Stemmadenia mollis, Montanoa tomentosa ssp. xanthiifolia y Luehea candida. El \%VI por familia deja ver la dominancia de Fabaceae $(\% \mathrm{VI}=18,64)$, seguida de Tiliaceae $(17,55)$, Burseraceae $(12,48)$ y Asteraceae $(12,41)$. Estas familias totalizan $61,08 \%$ del \%VI entre las 16 familias registradas (Tabla 2). También en número de especies la principal familia es Fabaceae, con un valor de 10. Le siguieron Asteraceae, Burseraceae y Tiliaceae con tres, cada una. El resto de familias contó con una o dos especies, lo cual es un caso de comprobación de la hipótesis de Rodríguez-Trejo (2014), relativa a la prevalencia de especies leguminosas en selvas bajas relacionadas con el fuego.

La selva baja incendiada y estudiada es rica en especies, pues en el diagnóstico para las tres asociaciones principales en las selvas bajas de toda la depresión central de Chiapas se refieren de 57 a 157 especies, y la dominancia de Fabaceae en número de especies se ha registrado en varias otras selvas bajas caducifolias del país, incluidas las de la depresión central (ROCHA; 
RAMÍREZ; GONZÁLEZ, 2010). La Tabla 3 muestra las principales especies supervivientes. Se da cuenta de 28 especies arbóreas que pueden tolerar o resistir fuego, pues tienen de dos a cuatro adaptaciones al fuego. El grosor de corteza varió entre 1 y $20 \mathrm{~mm}$. Scott et al. (2014) señalan que las cortezas gruesas proveen aislamiento térmico al cambium vascular. Entre las especies con grosores de hasta 10 a $20 \mathrm{~mm}$ y, por ende, con más posibilidades de adaptación al fuego por esta característica, están: Byrsonima crassifolia, 12 a $16 \mathrm{~mm}$, Cochlospermum vitifolium, 10 a $15 \mathrm{~mm}$, Liabum sublobatum, 10 a $13 \mathrm{~mm}$, Bursera simaruba y Bursera bipinnata (ambos con $10 \mathrm{~mm}$ ).

La regeneración por semilla no se halló en todos los sitios. Pero donde la hubo en algunos casos llegó a 1800 ha $^{-1}$. Consideramos que, al menos en parte, esta regeneración se presentó como en las zonas templadas, donde el fuego limpia de materiales orgánicos el piso forestal y permite el contacto de la semilla con el suelo mineral, facilitando el establecimiento de estas especies (MILLER, 2000), aunque diversas especies de este tipo de selvas pueden requerir de micrositios para su germinación y establecimiento. Las especies dominantes en la regeneración fueron Bauhinia ungulata y Acacia cornigera. Le siguieron Diphysa americana, Stemmadenia mollis, Heliocarpus reticulatus, Platymiscium dimorphandrum, Chrysophyllum mexicanum, Acacia milleriana y Luehea candida. Cinco de estas 9 especies son Fabaceae.

La latencia física se observa en muchas leguminosas, como las estudiadas (Platymiscium dimorphandrum, Bauhinia ungulata, Acacia milleriana, A. cornigera y otras) y es común en las Fabaceae. Scott et al. (2014), Baskin y Baskin (2001), Pausas (2012) y Rodríguez-Trejo (2014), señalan que la latencia física es una adaptación que permite a las semillas sobrevivir al paso por el tracto digestivo de fauna, ganado, y al fuego. Estos factores eliminan la latencia física, permiten la germinación y contribuyen a la regeneración.

Tabla 2 - Valores de importancia, selva baja con algunas especies secundarias o de selva mediana.

Table 2 - Importance values, tropical dry forest with some secondary or tropical rain forest species.

\begin{tabular}{|c|c|c|c|c|c|c|c|c|c|}
\hline Nombre científico (familia) & $\begin{array}{l}\text { Nombre } \\
\text { común }\end{array}$ & $\mathbf{F}$ & $\mathbf{F R}$ & $\underset{\left(\mathbf{m}^{2} \mathrm{ha} \mathrm{a}^{-1}\right)}{\mathrm{AB}}$ & DoR & $\begin{array}{c}\mathbf{n} \\
\left(\mathbf{h a}^{-1}\right)\end{array}$ & DeR & VI & $\% \mathrm{VI}$ \\
\hline Heliocarpus reticulatus Rose (Tiliaceae) ${ }^{*}$ & Namo & 15 & 7,21 & 2,91 & 18,93 & 228,57 & 9,52 & 35,66 & 11,89 \\
\hline $\begin{array}{l}\text { Bursera bipinnata (DC.) engl. (Bursera- } \\
\text { ceae) })^{*}\end{array}$ & Copal & 5 & 2,4 & 2,00 & 13,00 & 104,76 & 4,37 & 19,77 & 6,59 \\
\hline Liabum sublobatum Rob. (Asteraceae) ${ }^{*}$ & Gamuza & 4 & 1,92 & 1,54 & 10,00 & 185,71 & 7,74 & 19,66 & 6,55 \\
\hline $\begin{array}{l}\text { Stemmadenia mollis Benth. (Apocyna- } \\
\text { ceae) }{ }^{*}\end{array}$ & $\begin{array}{l}\text { Coyol de } \\
\text { cochi }\end{array}$ & 15 & 7,21 & 0,49 & 3,17 & 219,05 & 9,13 & 19,51 & 6,50 \\
\hline $\begin{array}{l}\text { Montanoa tomentosa ssp. xanthiifolia } \\
\text { (Sch. Bip.) B. L. Turner (Asteraceae) }\end{array}$ & Malacate & 12 & 5,77 & 0,32 & 2,09 & 233,33 & 9,72 & 17,58 & 5,86 \\
\hline Luehea candida (DC.) Mart. (Tiliaceae) & Cascabillo & 14 & 6,73 & 0,53 & 3,43 & 119,05 & 4,96 & 15,13 & 5,04 \\
\hline $\begin{array}{l}\text { Cochlospermum vitifolium (Willd.) } \\
\text { Spreng (Cochlospermaceae) }\end{array}$ & Pomposhuti & 9 & 4,33 & 1,07 & 6,97 & 61,90 & 2,58 & 13,88 & 4,63 \\
\hline $\begin{array}{l}\text { Platymiscium dimorphandrum (J. D. } \\
\text { Smith) Donn. Sm. (Fabaceae) }\end{array}$ & Hormiguillo & 12 & 5,77 & 0,45 & 2,95 & 109,52 & 4,56 & 13,29 & 4,43 \\
\hline Bauhinia ungulata L. (Fabaceae) & Pie de venado & 11 & 5,29 & 0,16 & 1,40 & 123,81 & 5,16 & 11,49 & 3,83 \\
\hline Acacia cornigera L. Willd. (Fabaceae) ${ }^{*}$ & Ishcanal & 8 & 3,85 & 0,32 & 2,08 & 104,76 & 4,37 & 10,29 & 3,43 \\
\hline Bursera sp. (Burseraceae) & Papelillo & 6 & 2,88 & 0,65 & 4,23 & 66,67 & 2,78 & 9,89 & 3,30 \\
\hline (varias especies) & Bejuco & 8 & 3,85 & 0,08 & 0,52 & 95,24 & 3,97 & 8,33 & 2,78 \\
\hline
\end{tabular}


Tabla 2 - Continuación...

Table 2 - Continuation...

\begin{tabular}{|c|c|c|c|c|c|c|c|c|c|}
\hline Nombre científico (familia) & $\begin{array}{l}\text { Nombre } \\
\text { común }\end{array}$ & $\mathbf{F}$ & FR & $\underset{\left(\mathbf{m}^{2} h \mathbf{a}^{-1)}\right.}{\mathrm{AB}}$ & DoR & $\begin{array}{c}\mathbf{n} \\
\left(\mathbf{h a}^{-1}\right)\end{array}$ & DeR & VI & $\% \mathrm{VI}$ \\
\hline $\begin{array}{l}\text { Bursera simaruba (L.) Sarg. (Bursera- } \\
\text { ceae)* }\end{array}$ & Mulato & 5 & 2,4 & 0,43 & 2,79 & 61,90 & 2,58 & 7,77 & 2,59 \\
\hline $\begin{array}{l}\text { Diphysa americana (Mill.) M. Sousa (Fa- } \\
\text { baceae) }\end{array}$ & Huachipilín & 7 & 3,37 & 0,27 & 1,77 & 52,38 & 2,18 & 7,32 & 2,44 \\
\hline Cedrela odorata L. (Meliaceae) & $\begin{array}{l}\text { Cedro } \\
\text { colorado }\end{array}$ & 8 & 3,85 & 0,24 & 1,58 & 42,86 & 1,79 & 7,21 & 2,40 \\
\hline $\begin{array}{l}\text { Byrsonima crassifolia L. (Kunth) (Malpi- } \\
\text { ghiaceae) }{ }^{*}\end{array}$ & Nanche & 4 & 1,92 & 0,45 & 2,92 & 28,57 & 1,19 & 6,03 & 2,01 \\
\hline $\begin{array}{l}\text { Chrysophylum mexicanum Brandegee } \\
\text { (Sapotaceae) }\end{array}$ & Caimito & 6 & 2,88 & 0,16 & 1,06 & 47,62 & 1,98 & 5,93 & 1,98 \\
\hline $\begin{array}{l}\text { Alibertia edulis (L. Rich.) A. Rich. (Ru- } \\
\text { biaceae) }\end{array}$ & Naranjillo & 2 & 0,96 & 0,21 & 1,33 & 19,05 & 0,79 & 3,09 & 1,03 \\
\hline $\begin{array}{l}\text { Lysiloma acapulcensis (Kunth.) Benth. } \\
\text { (Fabaceae) })^{*}\end{array}$ & Tepehuaje & 3 & 1,44 & 0,14 & 0,94 & 9,52 & 0,40 & 2,78 & 0,93 \\
\hline $\begin{array}{l}\text { Albizia caribaea (Urb.) Britton \& Rose } \\
\text { (Fabaceae) }\end{array}$ & Huaje blanco & 1 & 0,48 & 0,20 & 1,30 & 23,81 & 0,99 & 2,77 & 0,92 \\
\hline Lonchocarpus sp. (Fabaceae) & Mata buey & 2 & 0,96 & 0,17 & 1,08 & 9,52 & 0,40 & 2,44 & 0,81 \\
\hline Ateleia pterocarpa DC. (Fabaceae) & $\begin{array}{l}\text { Siente } \\
\text { peyejos }\end{array}$ & 3 & 1,44 & 0,05 & 0,34 & 14,29 & 0,60 & 2,38 & 0,79 \\
\hline $\begin{array}{l}\text { Rollinia membranacea Triana \& Planch. } \\
\text { (Annonaceae) }\end{array}$ & Anona & 3 & 1,44 & 0,05 & 0,29 & 14,29 & 0,60 & 2,33 & 0,78 \\
\hline Acacia milleriana Stand. (Fabaceae) ${ }^{*}$ & Quebracho & 3 & 1,44 & 0,03 & 0,23 & 14,29 & 0,60 & 2,26 & 0,75 \\
\hline Guazuma ulmifolia Lam. (Sterculiaceae) ${ }^{*}$ & $\begin{array}{l}\text { Cuaulote } \\
\text { negro }\end{array}$ & 1 & 0,48 & 0,12 & 0,78 & 14,29 & 0,60 & 1,86 & 0,62 \\
\hline Muntingia calabura L. (Tiliaceae)* & $\begin{array}{l}\text { Capulín } \\
\text { cimarrón }\end{array}$ & 1 & 0,48 & 0,18 & 1,18 & 4,76 & 0,20 & 1,85 & 0,62 \\
\hline Casearia nitida (L.) Jacq. (Flacourtiaceae) & $\begin{array}{l}\text { Café } \\
\text { cimarrón }\end{array}$ & 2 & 0,96 & 0,03 & 0,18 & 14,29 & 0,60 & 1,74 & 0,58 \\
\hline $\begin{array}{l}\text { Astronium graveolens Jacq. (Anacardia- } \\
\text { ceae) }\end{array}$ & Jocotillo & 1 & 0,48 & 0,10 & 0,62 & 4,76 & 0,20 & 1,30 & 0,43 \\
\hline $\begin{array}{l}\text { Ceiba aesculifolia (Kunth) Britton \& } \\
\text { Rose (Bombacaceae) }\end{array}$ & $\begin{array}{l}\text { Espina de } \\
\text { clavo }\end{array}$ & 1 & 0,48 & 0,03 & 0,22 & 14,29 & 0,60 & 1,29 & 0,43 \\
\hline Plumeria rubra L. (Apocynaceae) ${ }^{*}$ & Flor de mayo & 1 & 0,48 & 0,05 & 0,35 & 4,76 & 0,20 & 1,03 & 0,34 \\
\hline $\begin{array}{l}\text { Opuntia inaperta (Schott ex Griffiths) D. } \\
\text { R. Hunt (Cactaceae) }\end{array}$ & Nopal & 1 & 0,48 & 0,05 & 0,35 & 4,76 & 0,20 & 1,03 & 0,34 \\
\hline $\begin{array}{l}\text { Andira inermis (W. Wright) DC. (Faba- } \\
\text { ceae) }\end{array}$ & Lombricero & 1 & 0,48 & 0,04 & 0,24 & 4,76 & 0,20 & 0,92 & 0,31 \\
\hline
\end{tabular}


Tabla 2 - Conclusión...

Table 2 - Conclusion...

\begin{tabular}{lccccccccc}
\hline \multicolumn{1}{c}{ Nombre científico (familia) } & $\begin{array}{c}\text { Nombre } \\
\text { común }\end{array}$ & F & FR & $\begin{array}{c}\text { AB } \\
\left(\mathrm{m}^{2} \mathrm{ha}^{-1}\right)\end{array}$ & DoR & $\begin{array}{c}\mathbf{n} \\
\left(\mathrm{ha}^{-1}\right)\end{array}$ & DeR & VI & \%VI \\
\hline $\begin{array}{l}\text { Swietenia humilis Zuccarini (Meliaceae) } \\
\text { Ceiba pentandra }(\mathbf{L} \text {.) Gaertn. (Bombaca- }\end{array}$ & Caobilla & 1 & 0,48 & 0,01 & 0,09 & 4,76 & 0,20 & 0,77 & 0,26 \\
$\begin{array}{l}\text { ceae) } \\
\text { Desconocidas }\end{array}$ & Ceiba & 1 & 0,48 & 0,01 & 0,04 & 4,76 & 0,20 & 0,72 & 0,24 \\
\hline Sumas & & 31 & 14,9 & 1,83 & 11,91 & 333,33 & 13,89 & 40,70 & 13,57 \\
\hline
\end{tabular}

Donde: $\mathrm{F}=$ frecuencia; $\mathrm{FR}$ = frecuencia relativa $\mathrm{AB}=$ área basal; $\mathrm{DoR}=$ dominancia relativa $\mathrm{n}=$ número de individuos; DeR = densidad relativa; VI = valor de importancia; \%VI = porcentaje de valor de importancia. * Típica de selva baja.

Se identificaron diversos individuos con 2 a 6 rebrotes en más de 20 especies, como: Bauhinia ungulata, Liabum sublobatum, Montanoa tomentosa ssp. xanthiifolia, Bursera simaruba, Heliocarpus reticulatus, Albizia caribaea, Rollinia membranacea, Stemmadenia mollis, Chrysophyllum mexicanum, Luehea candida y Guazuma ulmifolia. Las especies de selvas están adaptadas para recuperarse mediante rebrotación de daños producidos por los vientos de huracanes y tormentas, pero muchas no rebrotan después de un incendio. Cuando ésta se presenta luego del fuego, como en este trabajo, la rebrotación también es un indicador de tolerancia al fuego.

Características como corteza gruesa, latencia física en la semilla, regeneración en áreas incendiadas, rebrotación (post-fuego), presencia de órganos rizomatosos y el que las especies sean representativas de vegetación secundaria, son indicativos de potencial adaptación al fuego (Tabla 3). Otra especie que no quedó dentro del muestreo, pero que ha sido observada en la zona y también muestra adaptaciones al fuego, es la parota (Enterolobium cyclocarpum (Jacq.) Griseb.). Su semilla tiene latencia física y su corteza es gruesa (20 a 30 $\mathrm{mm}$ ). Es una especie caducifolia, si bien se la ve tanto en selvas altas como medianas, pero con frecuencia en zonas perturbadas. El canelo (Calycophyllum candidissimum (Vahl) DC.), aunque típico de selvas medianas, tiene una corteza que puede alcanzar grosores de 15 $\mathrm{mm}$ y es deciduo. Parece tener resistencia al fuego, aunque no a incendios periódicos. La Figura 5 muestra algunos ejemplos de adaptaciones al fuego.

Entre las especies las hay típicas de perturbación, es decir, secundarias, como también abundan representantes de selvas bajas y algunas de selvas medianas. 15 de 20 especies cuyo estatus sucesional logró ser documentado bibliográficamente, son pioneras, secundarias iniciales o secundarias (Tabla 3), por lo cual dominan especies relacionadas con perturbaciones como el fuego. Además, dominan las especies caducifolias de selva baja (en estado de reposo resisten o toleran mejor al fuego) o bien de vegetación secundaria. En la Tabla 3 hay algunas especies de selva mediana, que con frecuencia tienen una corteza delgada, de unos cuantos milímetros de grosor. Se considera que aunque estas especies sobrevivieron a un incendio, no quiere decir que lo harían ante incendios repetidos, incluso espaciados en el tiempo. No obstante, desde 1952, Miranda (2015a) señalaba que muchas de las selvas bajas caducifolias de Chiapas tienen una composición alterada, producto de incendios y presión de ganado durante siglos, lo que en la selva estudiada logramos demostrar por la prevalencia de especies de etapas sucesionales iniciales y sus adaptaciones al fuego. Rocha, Ramírez y González (2010) señalan que en Chiapas sólo queda $>5 \%$ de la cubierta forestal original, y que de 40 a $56 \%$ de las especies en las selvas bajas de la Depresión Central son tardías sucesionalmente. Castillo, Halffter y Moreno (2008), refieren que la mayor parte de los paisajes de las selvas bajas caducifolias del país son mosaicos de tierras agrícolas, vegetación secundaria y parches con vegetación primaria relativamente conservada. 
Tabla 3 - Principales especies supervivientes año y medio después del incendio, con dos o más adaptaciones o adaptaciones potenciales al fuego.

Table 3 - Main surviving species, one year and a half after the forest fire, with two or more fire adaptations or potential fire adaptations.

\begin{tabular}{|c|c|c|c|c|c|c|}
\hline \multirow{2}{*}{ Nombre científico } & \multirow{2}{*}{ Etapa sucesional } & \multicolumn{5}{|c|}{ Adaptación potencial al fuego } \\
\hline & & CG & Reg & LF, Pi & Reb & $\mathbf{H}$ \\
\hline Stemmadenia mollis & S & & $\mathrm{X}$ & & $\mathrm{X}$ & \\
\hline Heliocarpus reticulatus & & & $\mathrm{X}$ & & $\mathrm{X}$ & \\
\hline Platymiscium dimorphandrum & & & $\mathrm{x}$ & $\mathrm{X}$ & & \\
\hline Diphysa americana & $\mathrm{T}, 10$ & & & $\mathrm{X}$ & & \\
\hline Montanoa tomentosa ssp. xanthiifolia & & & & & $\mathrm{x}$ & \\
\hline Bauhinia ungulata & $S, P, 10$ & & $\mathrm{x}$ & $\mathrm{X}$ & $\mathrm{x}$ & \\
\hline Cochlospermum vitifolium & $\mathrm{Si}, 3,6$ & $\mathrm{X} 1,6$ & & & & 6 \\
\hline Cedrela odorata & $\mathrm{Si}, 1,10$ & $\mathrm{X}, 6$ & $\mathrm{x}$ & & & 6 \\
\hline Acacia cornigera & $\mathrm{P}, 10$ & & $\mathrm{x}$ & $\mathrm{X}$ & & $\mathrm{X}, 8$ \\
\hline Acacia milleriana & & & $\mathrm{x}$ & $\mathrm{X}$ & $\mathrm{X}, \mathrm{a}$ & \\
\hline Rollinia membranacea & $\mathrm{S}, \mathrm{I}, 10$ & $\mathrm{X}, 6$ & $\mathrm{x}$ & & $\mathrm{x}$ & 6 \\
\hline Lysiloma acapulcensis & $\mathrm{P}, 10, \mathrm{Si}, 1,6,7$ & $\mathrm{X}, 6,7$ & & $\mathrm{x}$ & & $1,6,7$ \\
\hline Bursera bipinnata & $\mathrm{I}, \mathrm{Si}, 1,7,10$ & $\mathrm{x}$ & & & & 1 \\
\hline Bursera simaruba & $\mathrm{P}, \mathrm{Si}, 6,9,10$ & $\mathrm{X}, 2,6$ & & & $\mathrm{x}$ & $4,6,9, \mathrm{c}$ \\
\hline Byrsonima crassifolia & $\mathrm{Si}, 10, \mathrm{~b}$ & $\mathrm{X}, 1,6$ & $\mathrm{x}$ & $\mathrm{x}$ & $\mathrm{x}$ & $\mathrm{X}, 6$ \\
\hline Opuntia inaperta & & $\mathrm{x}$ & & & $\mathrm{x}$ & --- \\
\hline Spondias mombin & & 6 & & $\mathrm{Pi}$ & & 6 \\
\hline Plumeria rubra & $\mathrm{Si}, 1,3,10$ & 6 & & & & 6 \\
\hline Guazuma ulmifolia & $\mathrm{Si}, 3,6$ & & & & $\mathrm{x}$ & 6 \\
\hline Liabum sublobatum & & $\mathrm{x}$ & & & $\mathrm{x}$ & \\
\hline Chrysophyllum mexicanum & $\mathrm{Si}, 10$ & 3,6 & $\mathrm{x}$ & & $\mathrm{x}$ & $\mathrm{Pe}, 6$ \\
\hline Ceiba aesculifolia & $\mathrm{Si}, 6$ & 6 & & & & 6 \\
\hline Luehea candida & & & $\mathrm{x}$ & & $\mathrm{x}$ & \\
\hline Ateleia pterocarpa & $S$ & & & $\mathrm{x}$ & & \\
\hline Swietenia humilis & $\mathrm{T}, 1,5,7,10$ & 7 & $\mathrm{x}$ & & & 6 \\
\hline Albizia caribaea & & & & $\mathrm{X}$ & $\mathrm{x}$ & \\
\hline Lonchocarpus sp. & & & & $\mathrm{x}$ & & \\
\hline Ceiba pentandra & $S, 1,6,7$ & 6 & & & & 6 \\
\hline
\end{tabular}

Donde: $\mathrm{T}=$ tardía; $\mathrm{P}=$ pionera; $\mathrm{Si}=$ secundaria inicial; $\mathrm{I}=$ intermedia; $\mathrm{S}=$ secundaria; $\mathrm{CG}=$ corteza gruesa; Reg = regeneración por semilla en sitios incendiados; $\mathrm{LF}$ o $\mathrm{Pi}=$ semilla con latencia física o pireno; Reb = rebrotación; $\mathrm{H}=$ hábito caducifolio; $\mathrm{Pe}=$ perennifolia; $\mathrm{a}=$ puede emitir rebrotes a partir de órgano rizomatoso; $\mathrm{b}=$ típica de sabanas; $\mathrm{c}=$ generalmente; $\mathrm{X}=$ observaciones de campo del presente trabajo; Observaciones de la literatura: 1 = García y Linares (2013); 2 = Ochoa, Pérez y Jiménez (2008); 3 = Ochoa et al. (2008); 4= del Amo et al. (2009); 5 = Miranda (2015a; 2015b); 6 = Pennington y Sarukhán (2005); 7 = Guízar y Sánchez (1991); 8 = Dirzo, Martínez y Sinaca (1997); 9 = Dirzo y Sinaca (1997); 10 = Rocha, Ramírez y González (2010). 
Figura 5 - Algunas adaptaciones al fuego en árboles de la selva baja estudiada. A) Corteza gruesa de Diphysa americana B) Regeneración de pie de venado (Bauhinia ungulata). Cortezas gruesas de: C) Heliocarpus reticulatus, D) Lysiloma acapulcensis y E) Spondias mombin (fuera de los sitios de muestreo). F) Rebrote de rizoma (flecha) en Acacia milleriana.

Figure 5 - Some fire-traits in tree species of the tropical dry forest studied. A) Thick bark of Diphysa americana. B) Post-fire regeneration of "pie de venado" (Bauhinia ungulata). Thick barks of: C) Heliocarpus reticulatus, D) Lysiloma acapulcensis and E) Spondias mombin (out of the sampling sites). F) Resprout from rhizom, Acacia milleriana (arrow).

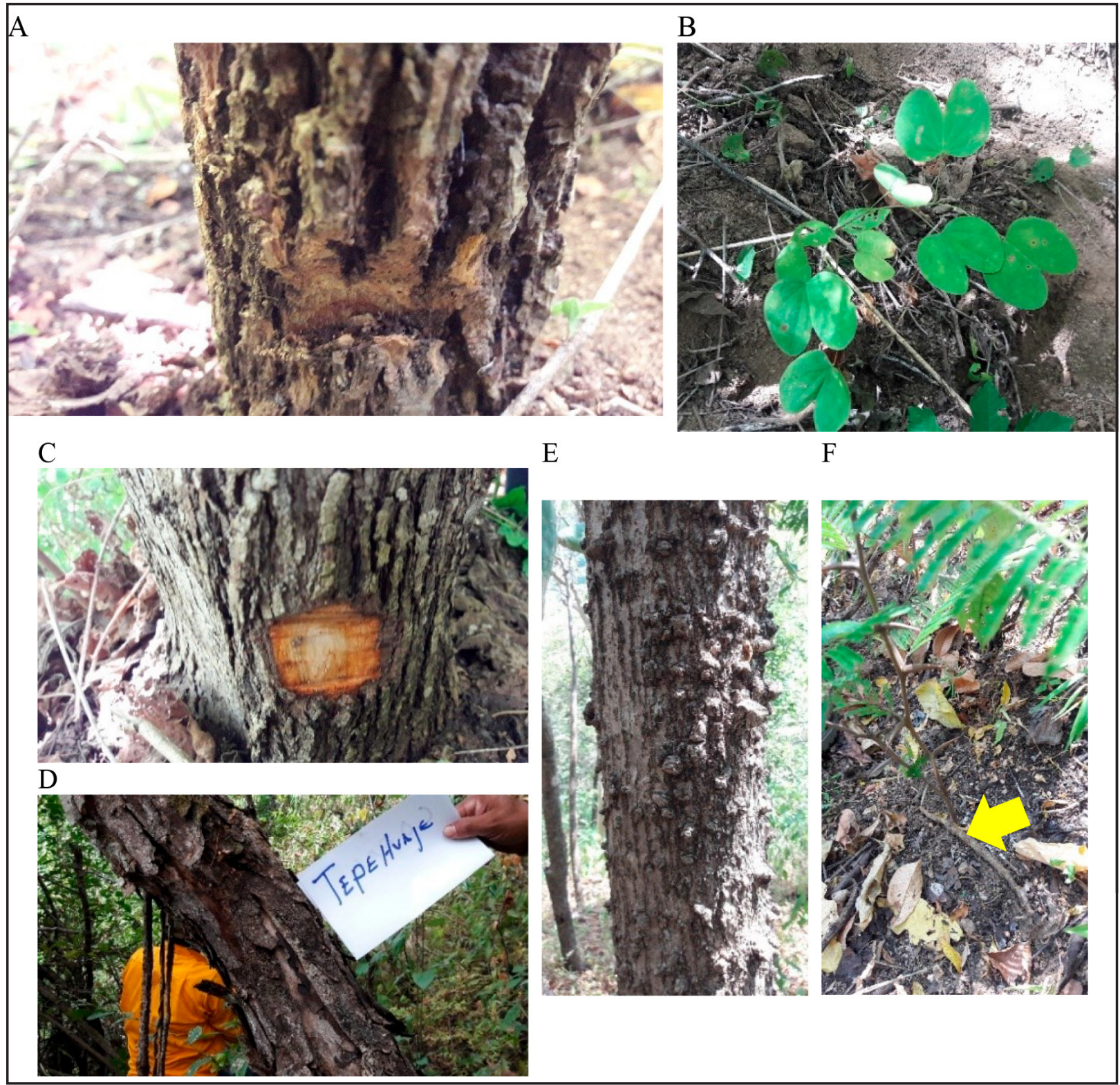

Fuente: Autores (2019)

Source: Authors (2019)

\section{Conclusiones}

Los modelos logísticos para estimar probabilidad de mortalidad y probabilidad de 
rebrotación, son una excelente herramienta para la evaluación de impactos potenciales de mortalidad del arbolado, y de su recuperación, en áreas incendiadas.

Asimismo, son una herramienta útil para mantener indicadores de severidad bajos al realizar quemas prescritas, de modo que la mortalidad sobre el arbolado adulto sea nula o mínima.

Las variables de los modelos confirman adaptaciones al fuego en Pinus oocarpa (corteza gruesa, recuperación de copa, rebrotación en la base, regeneración, altura a la base de la copa relacionada con poda natural) y características del arbolado que le confieren mayor resistencia ante el fuego (mayores altura y diámetros).

Aunque Pinus oocarpa es una especie adaptada al fuego, el incendio fue severo (altas cicatriz sobre el tronco y chamuscado de copa) y originó una elevada mortalidad, principalmente orientada a los individuos de pequeñas dimensiones.

La selva baja estudiada se encuentra perturbada y tiene algunas especies de selva mediana. Las 37 especies arbóreas registradas resistieron o toleraron el paso de un incendio forestal, de bajas intensidad y severidad, año y medio atrás. De ellas, 28 mostraron por lo menos dos adaptaciones o adaptaciones potenciales al fuego.

Además de las especies con mayor valor de importancia en esta selva, las más adaptadas al fuego fueron las pioneras, las secundarias y de la familia de las leguminosas.

A pesar de la alta supervivencia registrada a un año y medio del incendio, las especies muestran diferente magnitud en sus adaptaciones al fuego.

Es probable que de presentarse incendios más frecuentes que hasta ahora, irán eliminando a especies sucesionalmente avanzadas en selvas bajas, hasta sabanificarse o quedar como vegetación secundaria.

La prevalencia de especies adaptadas al fuego confirma que dicho factor ha operado sobre esta selva baja eliminando especies menos resistentes o tolerantes al fuego. Su existencia deja ver un régimen de fuego base (antropógena) que ha contribuido a definir y a mantener la composición actual, alterada, de la selva baja en el área de estudio. Se trata de una selva baja en la categoría de influenciada por el fuego.

\section{Agradecimientos}

Apoyo financiero o logístico: UACH, FMCN, USDA FS, US AID, Ayuntamiento de Villaflores, Biomasa, A. C. Ayuda en campo, al Sr. Arbert Toledo García.

\section{Referencias}

BASKIN, C. C.; BASKIN, J. M. Seeds. San Diego: Academic Press, 2001. 666 p.

CAstillo, C. G.; HAlfFter, G.; MOREnO, C. E. Primary and secondary vegetation patches as contributors to floristic diversity in a tropical deciduous forest landscape. Biodiversity and Conservation, Switzerland, v. 17, n. 7, p. 1701-1714, jun. 2008.

DEL AMO, R. S. et al. Germinación y manejo de especies forestales tropicales. México: Universidad Veracruzana, 2009. 122 p.

DIRZO, R.; MARTÍNEZ, R. I.; SINACA, C. S. Acacia cornigera. In: GONZÁLEZ, S. E.; DIRZO, R. R. C.; VOGT, R. C. (ed.). Historia Natural de Los Tuxtlas. México: UNAM, 1997. p. 87-88.

DIRZO, R.; SINACA, C. S. Bursera simaruba. In: GONZÁLEZ, S. E.; DIRZO, R.; VOGT, R. C. (ed.). Historia Natural de Los Tuxtlas. México: UNAM, 1997. p. 101-102.

GARCÍA, R. I.; LINARES, L. A. Árboles y arbustos de la cuenca del río Tepalcatepec. México: El Colegio de Michoacán; IPN, 2013. 394 p.

GUÍZAR, N. E.; SÁNCHEZ, V. A. Guía para el reconocimiento de los principales árboles del Alto Balsas. México: UACH, 1991. 203 p. 
HARDESTY, J.; MYERS, R. L.; FULKS, W. Fire, ecosystems and people: a preliminary assessment of fire as a global conservation issue. The George Wright Forum, [s. l.], v. 22, n. 4, p. 78-87. 2005.

HARTMANN, H. T. et al. Hartmann and Kester's plant propagation. $8^{\text {th }}$ ed. Boston: Prentice Hall, 2011. $915 \mathrm{p}$.

HOOD, S. M. et al. Evaluation of a post-fire tree mortality model for western US conifers. International Journal of Wildland Fire, Canberra, v. 16, n. 6, p. 679-689, 2007.

HOOD, S. M. et al. Fire and tree death: understanding and improving modeling of fire-induced tree mortality. Environmental Research Letters, [s. l.], v. 13, p. 1-14, 2018.

HOSMER JUNiOR, D. W.; LEMESHOW, S.; StURDIVANT, R. X. Applied logistic regression. $3^{\text {rd }}$ ed. Hoboken; New Jersey: John Wiley \& Sons, 2013. 528 p.

JUÁREZ B., J. E.; RODRÍGUEZ-TREJO, D. A.; MYERS, R. L. Fire tolerance of trees in an oak-pine forest at Chignahuapan, Puebla, Mexico. International Journal of Wildland Fire, Canberra, v. 21, n. 7, p. 873$881,2012$.

MILLER, M. Fire autecology. In: BROWN, J. K.; SMITH, J. K. (ed.). Wildland Fire in Ecosystems. Effects of Fire on Flora. Ogden: USDA Forest Service, 2000. v. 2, p. 9-34.

MIRANDA, F. La vegetación de Chiapas. Tuxtla Gutiérrez: UNICACH, 2015a. v. 1, 305 p.

MIRANDA, F. La vegetación de Chiapas. Tuxtla Gutiérrez: UNICACH, 2015b. v. 2, 381 p.

OCHOA, G. S.; PÉREZ, H. I.; JIMÉNEZ, P. N. C. Descripción de las especies de árboles más comunes de la sierra de Tenosique, Tabasco. México: Ecosur; Conacyt, 2008. 137 p.

OCHOA, G. S. et al. Manual de semillas de especies forestales de las montañas de Tenosique, Tabasco. México: Ecosur; Conacyt, 2008. 98 p.

ODUM, E. P.; BARRETT, G. W. Fundamentos de Ecología. México: CENCAGE Learning, 2006. 598 p.

PAUSAS, J. G. Incendios forestales. Madrid: CSIC; Catarata, 2012. 119 p.

PENNINGTON, T. D.; SARUKHÁN, K. J. Árboles tropicales de México. 3. ed. México: UNAM; FCE, 2005. $523 \mathrm{p}$.

ROCHA, L. A. G.; RAMÍREZ, M. N.; GONZÁLEZ, E. M. Riqueza y diversidad de árboles del bosque tropical caducifolio en la Depresión Central de Chiapas. Boletín de la Sociedad Botánica de México, Ciudad de México, n. 87, p. 89-103, 2010.

RODRÍGUEZ-TREJO, D. A. Educación e incendios forestales. México: Mundi Prensa; UACH, 2000. 201 p.

RODRÍGUEZ-TREJO, D. A. Fire regimes, fire ecology, and fire management in Mexico. Ambio, Netherlands, v. 37, n. 7/8, p. 548-556, 2008.

RODRÍGUEZ-TREJO, D. A. Incendios de vegetación. Su ecología, manejo e historia. México: C. P.; UACH; Semarnat; PPCIF; PNIP; Conafor; Conanp; ANCF; AMPF, 2014. 889 p.

RODRÍGUEZ-TREJO, D. A. et al. First year survival of Pinus hartwegii Lindl. in burned areas in different times. International Journal of Wildland Fire, Canberra, v. 16, n. 1, p. 54-62, 2007.

RODRÍGUEZ-TREJO, D. A.; FULÉ, P. Z. Fire ecology of Mexican pines and a fire management proposal. International Journal of Wildland Fire, Canberra, v. 12, n. 1, p. 23-37, 2003.

SCOTT, A. C. et al. Fire on earth: an introduction. Chichester: Wiley Blackwell, 2014. 413 p.

SHLISKY, A. et al. El fuego, los ecosistemas y la gente: amenazas y estrategias para la conservación global de la biodiversidad. Arlington: The Nature Conservancy, 2007. 20 p. (Informe Técnico Iniciativa Global para el Manejo del Fuego, 2007-02).

VILLEGAS, D. G. et al. Flora nectarífera y polinífera en el estado de Chiapas. Chiapas: SAGAR; Goberno Edo. Chiapas, 2000. 164 p. 